\title{
Implementing exercise therapy into primary health care
}

\section{Cameron Moore}

The benefits of exercise for the treatment and prevention of chronic disease are well-established and widespread across demographic profiles. ${ }^{1,2}$ In a recent metaepidemiological analysis, physical activity was observed to be as effective as pharmacological therapy - indicating that exercise and many drug interventions are similar in terms of cardiovascular disease and diabetes prevention, rehabilitation after stroke, and heart failure treatment. ${ }^{3}$ Furthermore, it is proposed that exercise therapy is often less expensive, targets multiple co-morbidities, and has fewer side-effects compared to pharmacological therapy.,5 Alarmingly, only $15 \%$ of Canadians are achieving the 150 minutes of moderate-to-vigorous physical activity per week as recommended by the Canadian Society for Exercise Physiology and the World Health Organization. ${ }^{6}$ While the clinical benefits of exercise are well established, it is evident that the best way to engage individuals in an active lifestyle remains largely unknown.

Primary health care is a gateway to the health care system that is accessed by patients across age, race, and gender categories. Therefore, primary care may provide an effective setting to implement exercise-promoting services targeting groups within the community who may benefit the most. For example, primary care-based initiatives like the Exercise is Medicine ${ }^{\circledR}$ campaign, which endeavours to integrate exercise therapy into the health care system by making patients' physical activity levels a standard "vital sign" question, are gaining increased traction in Canada and the United States. ${ }^{7}$ However, exercise therapy has not been sufficiently adopted into the primary care environment; providing physicians and other primary care providers with the resources to counsel or refer their patients to appropriate physical activity therapy must be addressed by national and provincial policy reforms.

Recently, our research group undertook an environmental scan of exercise services offered by the 186 Family Health
Teams in Ontario. A Family Health Team is a framework where family physicians work alongside a team of health care professionals to offer comprehensive interprofessional primary health care to over 2.86 million Ontarians. In addition, the Ontario Government provides health promotion funding to approximately a third of Family Health Teams, including 48.5 full-time equivalent health promoter positions to facilitate chronic disease prevention and management programs (e.g., smoking cessation, healthy eating, exercise therapy, etc.) [Personal communication with a representative from the Ontario Ministry of Health and Long-term Care. 2014 Feb 21]. Family Health Teams only serve a fraction of Ontarians; however, because of the availability of health promotion resources and the multi-disciplinary environment, it is proposed that Family Health Teams should be at the forefront of exercise therapy delivery.

The sample of Family Health Teams surveyed in the environmental scan indicated $50 \%$ of Family Health Teams offered an exercise service. The availability of exercise services varied depending on the individual site, and services were often restricted to specific conditions or needs rather than available to the majority of patients. Only half of the Family Health Teams offering an exercise service employed a health promoter to provide exercise therapy, and the individuals providing exercise therapy did not always have qualifications associated with expertise in evidence-informed, patient-centered exercise prescription. The variability in exercise service delivery suggests that decisions about program structure and content are made locally, and may not be based on guidelines regarding what interventions are effective for changing behaviour or improving health outcomes in primary care.

Given the established health benefits of exercise therapy, all individuals, regardless of health status or location, would benefit from access to safe and effective exercise 
c services. Therefore, policy changes could be used to guide exercise therapy delivered in primary care. First, there are many evidence-informed, population-specific physical activity guidelines provided by national bodies (e.g., Canadian Society for Exercise Physiology) and stakeholder groups (e.g., Osteoporosis Canada) that could be used to standardize exercise services. Secondly, the primary health care system could benefit from the increased integration of health professionals with an in-depth understanding of exercise guidelines, contraindications to exercise, and behaviour change strategies to encourage exercise engagement. ${ }^{7}$ Kinesiologists for example, who are working under a newly accredited professional designation in Ontario, have an exercise-specific scope of practice, and the opportunity to acquire skills suited to offering safe and effective exercise therapy to diverse patient populations. Evidence has shown that the integration of a health promoter to provide exercise therapy in a primary care environment not only removes the responsibility of exercise prescription from the physician, but does so at an enticing cost-benefit ratio. ${ }^{5,9}$

In conclusion, the evidence base supporting exercise as medicine has reached a tipping point where primary care policy must be reformed to incorporate the widespread implementation of physical activity counselling and services. A standardized model, where physicians or other allied health professionals can assess physical activity needs and refer patients to a health promoter to deliver appropriate exercise recommendations and facilitate engagement in exercise should be considered. Such a model may serve to maximize the use of scarce health professional resources, reduce health care costs, and result in an overall improvement in public health.

\section{References}

1. Warburton DE, Nicol CW, Bredin SS. Health benefits of physical activity: The evidence. CMAJ. 2006;174(6):801-9.

2. Katzmarzyk PT, Gledhill N, Shephard RJ. The economic burden of physical inactivity in Canada. CMAJ. 2000;163(11):1435-40.

3. Huseyin Naci, John P A loannidis. Comparative effectiveness of exercise and drug interventions on mortality outcomes: Metaepidemiological study. BMJ. 2013; 347-f5577.

4. Knowler WC, Barrett-Connor E, Fowler SE, Hamman RF, Lachin JM, Walker $E A$, et al. Reduction in the incidence of type 2 diabetes with lifestyle intervention or metformin. N Engl J Med. 2002;346(6):393-403.

5. Hogg WE, Zhao X, Angus D, Fortier M, Zhong J, O'Sullivan T, et al. The cost of integrating a physical activity counselor in the primary health care team. J Am Board Fam Med. 2012;25(2):250-2.

6. Woodcock J, Franco OH, Orsini N, Roberts I. Non-vigorous physical activity and all-cause mortality: systematic review and meta-analysis of cohort studies. Int J Epidemiol. 2011 Feb;40(1):121-38.

7. American College of Sports Medicine. Exercise is Medicine ${ }^{\circledR}$ [Internet]. Indianapolis; 2008 [last update unknown; cited 2014 Jan 15]. Available from: http://exerciseismedicine.org

8. Warburton DE, Charlesworth SA, Foulds HJ, McKenzie DC, Shephard RJ, Bredin SS. Qualified exercise professionals: Best practice for work with clinical populations. Can Fam Physician. 2013;59(7):759-61.

9. Tulloch $\mathrm{H}$, Fortier $\mathrm{M}$, Hogg W. Physical activity counseling in primary care: who has and who should be counseling? Patient Educ Couns. 2006;64(13):6-20.

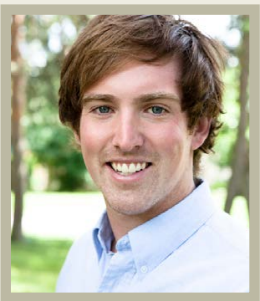

\section{Cameron Moore}

Cameron Moore has recently completed his M.Sc. in Kinesiology at the University of Waterloo and is a member of the Neuroengineering and Therapeutics Team at the University Health Network - Toronto Rehab. His research examines the impact of leg muscle size and quality on metabolic health over the lifespan of individuals with long-term spinal cord injury. 